\title{
STORYTELLING FOR RECREATING OUR SELVES: ZENETIC COMPUTER
}

\author{
Naoko Tosa \\ Massachusetts Institute of Technology /JST, \\ N52-390, 265 Massachusetts Ave. Cambridge, MA USA, : Japan Science Technology \\ Coporation "Interaction \& Intelligence" 2-2-2, Hikaridai, Seika-cho, Soraku-gun, Kyoto \\ 619-0288, Japan, \\ mail:naoko@mit.edu
}

Abstract: We present an interactive storytelling system that aims to help us "recreate" our conscious selves by calling on traditional Japanese concepts and media. "Recreating our selves" means the process of reconciling our conscious 'daily self' and our 'hidden self'. This requires deep stimulations which are difficult to achieve through conventional logic based interactions. Users create, enter and dynamically interact with a virtual world expressed as 3D "Sansui" ink painting, encountering fragments of stories and ambiguous provocations. The user physically interacts with the system through various media including a Sumie (ink painting), a rake in a Zen rock garden, touching screen images, drawing or clapping hands. The interaction system includes a dynamical chaos engine which is used to couple activity of the user to the generation of high dimensional context and evolution of the storytelling.

Key words:

\section{INTRODUCTION}

We have developed an interactive storytelling system that aims to help us "recreate" our conscious selves by calling on Buddhist principles, Asian philosophy, and traditional Japanese culture through the inspirational media of traditional ink paintings, kimono and haiku. "Recreating ourselves" means the process of making the consciousness of our 'daily self' meet that of our "hidden self'. through stimulation of activity deep within us. It is difficult to achieve this through traditional logic-based interactions. Our system is a new 
approach which incorporates traditional media and methods in an interactive computer system. The interactive storytelling stimulates deep imagination and allows users to develop connections between their hidden selves, full of imagination and creative energy, and their daily conscious selves, which directly interpret the ambient reality [1].

\section{PHILOSOPHY OF ZENETIC COMPUTER}

The user creates a virtual world by manipulating 3D images of Asian sansui ink painting on a computer display with an intuitive and enjoyable interface tool. These images, which typically symbolize nature and philosophical precepts, provide a dramatic departure from our view of daily experience. This awakens us from our daily consciousness and gives free reign to subconscious imagination[2]. Based on the user's sansui design, the system infers his or her internal consciousness and generates a story that the user can 'enter' via the computer display. This story further shakes the user's consciousness. This is not a complete story, such as those in the movies or novels, but fragments of short stories. Experiencing these episodic stories makes users feel uneasy and arouses their subconscious desire to construct a whole story by linking the fragments. In each of these inchoate stories, the system stimulates interaction through Zen dialogue or haiku as a form of allegorical communication. The user is asked questions that do not have "correct" answers. He or she is forced to deal with these ambiguous provocations while subconsciously struggling to answer the questions.

This subconscious effort inspires the user to find ways of linking the stories into an original whole. The user responds to objects presented by the interactive system, whether a graphic image or a provocative statement, by manipulating input media, such as a virtual calligraphy brush or rake of a Zen rock garden, on-screen images, or simply clapping hands. Coupled with the subconscious effort exerted to link the fragmentary stories, these user interactions decrease the gap between daily self and hidden self. This process of bringing our selves together is called MA-Interaction; ma is a Japanese concept that stresses the ephemeral quality of experience. In the final phase, the user has a dialogue with a "bull," which is used as a metaphor of our hidden self in Zen Buddhism. Through this dialogue, users experience a virtual unification of their daily self and their unconscious self into a recreated conscious self. 


\section{TECHNICAL REALIZATION}

Key technologies used to realize the system include a digital 3D sansui ink-painting engine which allows the users themselves to compose an ink painting to enter, a neural network engine which classifies the user's 'hidden personality' revealed in the ink painting into Buddhist Goun categories, and a dynamical chaos engine which is injected with signals from Goun categories and other user actions to generate high dimensional data for the context and evolution of the storytelling. The following are the main components of the system structure.

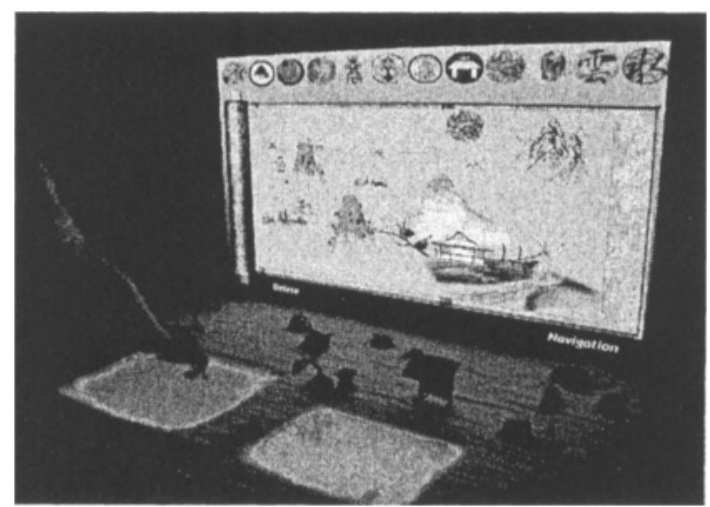

Figure 1. User make ink painting by ZENetic Computer

\subsection{Software Integration [3]}

The flow of the system is as follows:

1) User makes 3D Sansui ink-painting picture by manipulating symbolic icons

2) User's hidden self is classified into Goun categories.

3) User enters the Sansui picture and a journey begins. Haiku is used to generate story fragments that are presented in Sansui.

4) User experiences various stages of MA-Interaction (User may experience Steps 3 and 4 several times).

5) Finally, the Ten Bulls Story Interaction takes place. 


\subsection{Hardware Structure}

Figure 1 shows the overall hardware structure of the Zenetic Computer System.

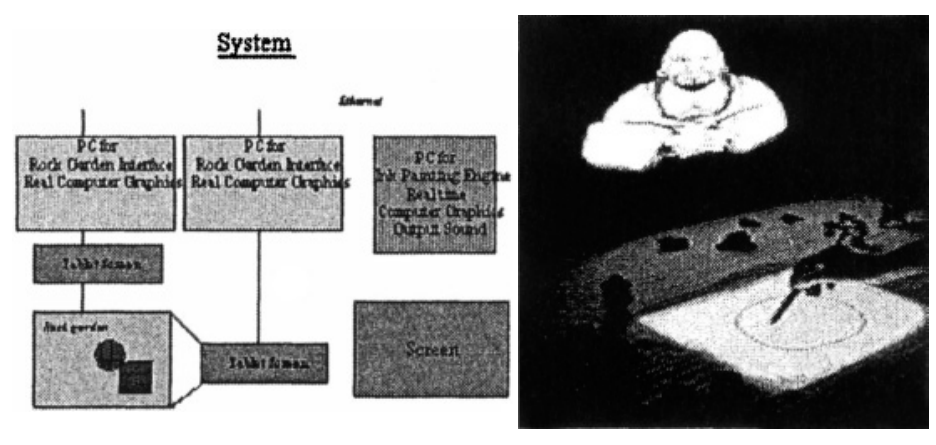

Figure 2. ZENeticComputer System

Figure 3. ZEN dialogue Interaction

\subsection{D Sansui Ink-painting engine}

A key part of the system is the user interaction with a digital 3D inkpainting engine. Depending on how users compose their initial ink-painting, the system classifies their intrinsic personality using a neural network. The personality corresponds to a point in a Goun space. Goun is a categorization from Buddhism based on the view that five basic spirits and materials make up the world. The five categories of personality based on goun can be summarized as follows.

a) 色 Shiki is how nature and materials actually exist.

b) 受 $J y u$ is the intuitive impression.

c) 想 So is the perceived image.

d) 行 Gyo is the process of mind that activates your behavior.

e) 識 Shiki is the deep mental process that lies behind all of the above processes

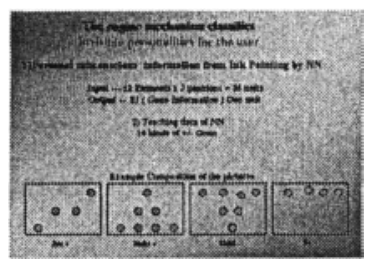

Figure 4. Examples of neural-net classification of the composition of pictures created by a user into Buddhist Goun categories corresponding to "hidden self'. 
User data is also obtained at later times from various interactions between the user and the system, and used to determine a pseudo Goun personality. Depending on how the user is affected by the evolving story, the pseudo Goun personality may differ from the intrinsic (=hidden) personality. Conversely, the difference between the pseudo personality and the intrinsic personality will affect the evolving story via an engine, called a chaos engine.

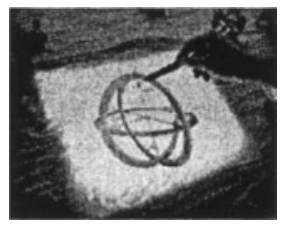

Figure 5. Compass for navigation in your ink painting world.

\subsection{Storytelling generated by chaos engine}

A dynamical chaos engine is used to couple activity of the user, via the difference between the pseudo personality and the intrinsic personality, to the generation of high dimensional context and evolution of the storytelling. The chaos engine consists of three dynamic components, which we call agents. We name the three agents, User, Target and Master. The agents each have internal chaotic dynamics, and also move around in Goun space. The three agents are coupled so that there is an interplay between their motions in the Goun space and the synchronization [4] of their internal dynamics. The transient dynamics of the chaos engine are sampled and used to create the sounds and images experienced by the user, and also to control the evolution of the story.

In the current implementation of the chaos engine for the ZENetic computer, the position of the User agent corresponds to the user's pseudo personality, and the position of the Target agent corresponds to the momentary view of the user's pseudo personality obtained from the latest user interaction. The User agent starts at the position of the intrinsic personality and tends to move toward the position of the Target agent. The User agent is coupled to the Target via the Master in such away that if there is no interference from the Master, the User tends to synchronize to the Target and move toward the Target position, so that the User and Target become identical. On the other hand, if there is interference from the Master, it is more difficult for the User to synchronize with the Target, and so less likely that the User will reach the Target. The strength of the Master's interference depends inversely on the distance between the pseudo personality and the hidden personality - the smaller the distance, the stronger 
the influence of the Master, and hence the more difficult it is for the User to synchronize and merge with the Target.

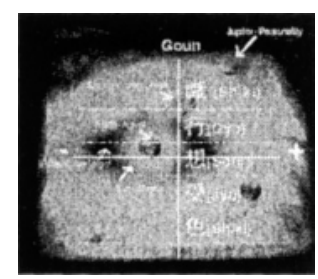

Figure 6. Visualization of your own Chaos engine in ZENetic Computer ZENetic Computer.

\section{CONCLUSIONS AND FUTURE WORK}

Real-time interaction with individual consciousness and subconscious is a long-term challenge for computer systems. Interactive storytelling is a frontier which allows us to explore this challenge. Science says that human consciousness may have a chaotic nature. By incorporating chaotic mechanisms, our system aims to provide a rich and dynamic interaction which entangles the conscious and subconscious. Responses to questionnaires from users who have experienced the ZENetic Computer show that they tend to feel relaxed and stimulated in a way that they had never felt before. Both English and Japanese versions have been developed. This system will be exhibited at the MIT Museum, KODAIJI - ZEN Temple (Kyoto, Japan) and around the world. [5]

\section{ACKNOWLEDGMENTS}

This research was supported in part by the Telecommunications Advancement Organization of Japan. Thanks to Dr. Peter Davis for helping to design and tune the chaos engine.

\section{REFERENCES}

1. S. Matsuoka,"The Science of the beauties of nature," Shunjusha (1994) (In Japanese)

2. S. Matsuoka, "Sansui Thought" Gogatsu shobo (2003) (In Japanese)

3. N. Tosa, Chapter19: Expression of emotion unconsciousness with art and technology, "AFFECTIVE MINDS," ELSEVIER, pp. 183-201 (2000)

4. Y. Liu, P. Davis, "Dual synchronization of chaos", Physical Review E, 61 pp. R2176R2179 (2000)

5. http://web.mit.edu/ naoko/ 